\title{
Low linkage disequilibrium in wild Anopheles gambiae s.l. populations
}

Caroline Harris $^{1 *}$, François Rousset ${ }^{2}$, Isabelle Morlais ${ }^{1}$, Didier Fontenille ${ }^{1}$, Anna Cohuet $^{3}$

\begin{abstract}
Background: In the malaria vector Anopheles gambiae, understanding diversity in natural populations and genetic components of important phenotypes such as resistance to malaria infection is crucial for developing new malaria transmission blocking strategies. The design and interpretation of many studies here depends critically on Linkage disequilibrium (LD). For example in association studies, LD determines the density of Single Nucleotide Polymorphisms (SNPs) to be genotyped to represent the majority of the genomic information. Here, we aim to determine LD in wild An. gambiae s.l. populations in 4 genes potentially involved in mosquito immune responses against pathogens (Gambicin, NOS, REL2 and FBN9) using previously published and newly generated sequences.

Results: The level of LD between SNP pairs in cloned sequences of each gene was determined for 7 species (or incipient species) of the An. gambiae complex. In all tested genes and species, LD between SNPs was low: even at short distances $(<200 \mathrm{bp})$, most SNP pairs gave an $r^{2}<0.3$. Mean $r^{2}$ ranged from 0.073 to 0.766 . In most genes and species LD decayed very rapidly with increasing inter-marker distance.

Conclusions: These results are of great interest for the development of large scale polymorphism studies, as LD generally falls below any useful limit. It indicates that very fine scale SNP detection will be required to give an overall view of genome-wide polymorphism. Perhaps a more feasible approach to genome wide association studies is to use targeted approaches using candidate gene selection to detect association to phenotypes of interest.
\end{abstract}

\section{Background}

When alleles at different loci appear together in individuals more often than would be expected by chance they are said to be in Linkage Disequilibrium (LD) [1]. $\mathrm{LD}$ is an indicator of the rate of recombination events between markers during meiosis. In addition to nucleotide distance, the effective recombination rate can be affected by numerous forces in natural populations such as selection that maintains certain allele associations (epistasis), genetic drift, population structure and demographic changes. Non random association between variants has recently become the focus of intense study in the hope that it might facilitate the mapping of complex trait loci through genome wide association studies (GWAS). Indeed, recent progress in the technological

\footnotetext{
* Correspondence: Caroline.Harris@ird.fr

'Laboratoire de Lutte Contre les Insectes Nuisibles, Unité de Recherche 016Institut de Recherche pour le Développement, B.P. 64501, 34394 Montpellier Cedex 5, France

Full list of author information is available at the end of the article
}

ability to genotype genetic variation [2] opens promising possibilities for identification of variants linked to phenotypes of interest. However, the ability to detect association critically depends on the extent of LD between causative alleles and surrounding markers. When LD extends over large genomic regions, there is a higher chance of finding association with the drawback that the potentially long physical distance between the gene of interest and an associated marker can make causative gene identification tedious. On the other hand, limited LD requires a much denser marker map to find associations, but, when found, identifying the causative allele is expected to be more straightforward [3].

The Anopheles gambiae species complex is of great interest due to its substantial role in malaria transmission. The two molecular forms or incipient species, $M$ and S, of Anopheles gambiae s.s. and Anopheles arabiensis are major vectors throughout sub Saharan Africa. The other species of the complex can have high local importance in malaria transmission or more minor roles 
depending on their biology and distribution [4]. Due to its epidemiological importance, several genomes $[5,6]$ and genes of interest [e.g. [7-10]] have been sequenced in An. gambiae s.s., providing a large data set of SNPs (Single Nucleotide Polymorphisms). Many of these variants are shared with the other members of the complex $[7,8]$. Today, the development of large scale genotyping tools makes the implementation of GWAS in An. gambiae s.s. and possibly the other members very realistic. However, to date little is known about LD in natural populations. In An. gambiae s.s., analysis of SNPs in six genes located in and around the 2La chromosomal inversion revealed LD over more than $30 \mathrm{Mbp}$ [11]. Strong LD was also detected within and between centromeric regions of the two incipient species $\mathrm{M}$ and $\mathrm{S}$ of An. gambiae s.s.[12]. This LD likely indicated subdivided populations adapted to different environments or incipient species, indeed, limited recombination rates in these chromosomal areas are hypothesized to be involved in environmental adaptation by maintaining combinations of alleles adapted to given conditions [13] and/or implicated in the speciation process through cause or consequence [12]. In other regions of the genome, very little data is available to our knowledge in natural populations; LD was measured only to exclude redundancy of markers or Wahlund effect in population genetic studies and rarely showed linked markers [e.g. [14-16]]. For instance, Lehmann et al. [16] tested the LD between microsatellite markers in several populations and observed only few pairwise combinations in significant $\mathrm{LD}$, corresponding to the proportion of the tests expected to be significant by chance alone. This indicated random association between the tested markers spread throughout the genome of An. gambiae s.s., however this is only mildly informative for short distance LD and the density of markers to be used in association studies.

In this study, we aimed to determine LD in An. gambiae s.l. between short range markers. LD data will be particularly informative for mapping genes involved in susceptibility to infection. In this context, immune related genes are primary candidates and rates of LD decay in these genes will be crucial in determining the density of markers to be used in such association studies. Four immune related genes were selected, namely, Gambicin, NOS, REL2 and FBN9, representative of different functions in the immune response and located in different chromosomal regions. Gambicin codes for an important antimicrobial peptide, which currently has no known specificity to Plasmodium [17]. NOS codes for Nitric oxide synthase, which markedly controls the infection level of Plasmodium in Anopheles $[18,19]$ but is likely to play different roles depending on parasite species [20]. REL2 is an NF-KappaB-like transcription factor that affects the development of Plasmodium in Anopheles in a conserved manner across several species of parasite and mosquito [21]. FBN9 codes for a Fibrinogen-domain protein whose silencing increases $P$. falciparum development [17].

For each gene, sequences of seven species of the $A n$. gambiae complex were analyzed and LD between polymorphic sites measured to estimate the extent of information given by genotyping a single polymorphism. We used previously published sequences [8] for 6 of the species and produced new data for a population of An. gambiae s.s M molecular form from Cameroon. Phased sequences were used to provide accurate haplotype data for estimating LD. To our knowledge, this is the first study on LD in An. gambiae based on phased sequences, allowing powerful analysis of LD decay over short distances. Moreover it is informative across almost all species members of the An. gambiae complex and focuses on genes of interest for future association studies.

\section{Methods}

Phased sequences, resulting from cloned DNA, were previously published for the genes Gambicin (AGAP008645), NOS (AGAP008255), REL2 (AGAP006747) and FBN9 (AGAP011197) for 5 to 14 field collected individuals of An. gambiae $\mathrm{S}$ form, An. arabiensis, An. melas, An. merus, An. quadriannulatus A and An. bwambae [8]. No evidence for positive selection was identified in these genes. Here, we provide data for the corresponding gene fragments for 16 individuals of An. gambiae M form collected in Simbock in South Cameroon.

Genes were localized on the An. gambiae s.s. genome using Vectorbase [20], and their positions relative to polymorphic chromosomal inversions determined [22]. Gambicin is positioned in subdivision $31 \mathrm{~A}$ on chromosome $3 \mathrm{R}$, where the inversions $3 \mathrm{Ra}$ in $\mathrm{An}$. arabiensis and $3 R e$ in $A n$. melas are known to be polymorphic. $N O S$ is in the subdivision 30A on chromosome 3R, it is located in polymorphic inversions $3 \mathrm{Ra}$ in An. arabiensis, $3 \mathrm{Rb}$ in $A n$. bwambae and 3Re in An. melas. FBN9 is in subdivision $42 \mathrm{~A}$ on chromosome $3 \mathrm{~L}$, where no polymorphic inversions are known within each species. REL2 is in subdivision 25D on chromosome $2 \mathrm{~L}$, here only the rare inversion $2 \mathrm{Ld}$ is known to be polymorphic in An. arabiensis but was not observed in Cameroon where the specimens were collected (Frederic Simard, personal communication).

To sequence An. gambiae M form individuals, DNA was extracted from mosquitoes as previously described [9]. Species and molecular forms were determined by diagnostic PCR [23]. Gene amplifications were carried out using the external primers and conditions previously published [8]. PCR products were cloned using 
the TOPO TA Cloning Kit for Sequencing (Invitrogen) and a minimum of five transformed colonies selected for sequencing. Inserts were amplified by PCR from the plasmid using the same external primers/conditions and were sequenced in both directions using the Big Dye Terminator v3.1 Sequencing Kit (Applied Biosystems). Sequences were verified by eye in SeqScape (Applied Biosystems) and aligned in Mega v.4.0.2 [24]. High Fidelity Taq was used (Platinum ${ }^{\circ}$ Taq DNA Polymerase High Fidelity, Invitrogen) in all PCRs to limit miss-incorporations. Sequences have been submitted to GenBank under accession numbers GU990095 to GU990222.

Analyses of polymorphism were carried out on all the previously published and new An. gambiae M form sequences using DNAsp v.5.10 [25]. LD was measured as $r$ for each pair of SNPs in each gene and species, significance $(\mathrm{P}<0.05)$ was tested using Fisher's exact test in DNAsp and the Bonferroni procedure applied to correct for multiple testing. $r$ was converted to $r^{2}$ and graphs of $\mathrm{r}^{2}$ relative to the distance between pairs of polymorphic sites plotted in R v.2.10.0 [26]. LD decay lines were modeled again in $\mathrm{R}$ by fitting data to the expectations of a simple population genetic model [27] using the non-linear least squares method, and to a nonparametric model. The non-linear fit to expectations failed (nls function in $\mathrm{R}$ failed to converge) for some species/locus combinations because the observed patterns deviated too much from the expectations, in particular, due to them showing an increase in LD at short distances, or, LD measures that exceed the maximum allowed by the analytical expression [[27] p.77]. Hence, only the results of the nonparametric model will be presented. The non-parametric model was a "generalized additive model" where the fit is a linear combination of observed values, whose coefficients are given by a cubic spline and the degree of smoothing determined by generalized cross-validation [28]. This computation was performed using the gam function from the mgcv package in $\mathrm{R}$.

Moreover, in order to detect LD haploblocks, grid plots were generated using Haploview 4.2 [29] for the $A n$. gambiae $\mathrm{M}$ sequences for each of the four tested genes.

To calculate whether there are significant differences in LD between genes and species, mean values of the $\mathrm{r}^{2}$ estimates were considered in each locus and species. The 7 values per locus (corresponding to the 7 species) or 4 values per species (corresponding to the 4 loci) were listed and compared by the Wilcoxon test by pair of loci or pair of species. As the numbers of values included in the tests were critically low, we attempted to increase the power of the tests by generating 4 values per locus and species. For that purpose, the sequences were cut into 4 segments of equal length and mean $r^{2}$ calculated for each segment to give independent values (4 mean values for one gene in one species). The 4 mean $r^{2}$ values were listed and grouped either according to gene (4 values for each of the 7 species resulting in 28 values in each list) or species (4 values for each of the 4 loci resulting in 16 values in each list) and the Wilcoxon test calculated in $\mathrm{R}$ to look for significant differences between groups (either each gene or each species).

\section{Results and discussion}

Sequences for 16 individuals of the An. gambiae M form were analyzed for the four genes. The polymorphism parameters are given in Table 1 . Genetic diversity (Pi) in An. gambiae M form, ranging from 0.0099 to 0.0253 , has comparable values to other species of the complex [8] and to other immunity genes [30-32]. As expected, the inclusion of introns increases the diversity compared to studies including only coding regions [7]. The high number of variant sites in An. gambiae $\mathrm{M}$ form and other species allowed a large number of pairwise measures of LD in almost all species and tested genes (Table 2). A very small proportion of pairwise measures was significantly in LD, although An. gambiae M revealed slightly more significant tests than other species, most likely a consequence of the bigger sample size (Table 2). Only REL2 in An. bwambae was not polymorphic. Plots of $r^{2}$ as a function of nucleotide distance are presented in Figure 1 for all species and genes and grid plots for An. gambiae M in Figure 2. Mean values of $\mathrm{r}^{2}$ (using whole sequences) for each gene and species are shown in Table 2.

Mean $\mathrm{r}^{2}$ values in genes and species range from 0.073 to 0.766 , with most falling below 0.3 . Only three mean values exceed this threshold; they correspond to NOS in

Table 1 Sequence and polymorphism parameters of the four amplified immunity genes in An. gambiae M form

\begin{tabular}{|c|c|c|c|c|c|c|c|}
\hline \multirow[t]{2}{*}{ Gene } & \multirow{2}{*}{$\begin{array}{l}\text { Number of } \\
\text { individuals }\end{array}$} & \multirow{2}{*}{$\begin{array}{l}\text { Length of sequences } \\
\text { analyzed (bp) }\end{array}$} & \multirow{2}{*}{$\begin{array}{l}\text { Number of } \\
\text { haplotypes }\end{array}$} & \multirow{2}{*}{$\begin{array}{l}\text { Number of polymorphic } \\
\text { sites }\end{array}$} & \multicolumn{3}{|c|}{ Nucleotide diversity (Pi) } \\
\hline & & & & & Total & Synonymous & Non-syn. \\
\hline Gambicin & 16 & 1108 & 24 & 105 & 0.0253 & 0.0155 & 0.0032 \\
\hline NOS & 16 & 1238 & 19 & 99 & 0.0247 & 0.0383 & 0.0009 \\
\hline REL2 & 16 & 834 & 21 & 40 & 0.0099 & 0.0220 & 0.0039 \\
\hline FBN9 & 16 & 735 & 18 & 53 & 0.0159 & 0.0664 & 0.0021 \\
\hline
\end{tabular}


Table 2 Number of pairwise measures of $r^{2}$ and number of significant pairwise measures using the Fisher's exact test after Bonferroni correction in brackets (all in bold) and mean $\mathbf{r}^{2}$ (standard deviation in brackets)

\begin{tabular}{|c|c|c|c|c|c|c|c|}
\hline Gene & An. gambiae $\mathrm{M}$ form & An. gambiae $\mathrm{S}$ form & An. arabiensis & An. bwambae & An. melas & An. merus & An. quadriannulatus \\
\hline \multirow[t]{2}{*}{ Gambicin } & $n=16$ & $n=5$ & $\mathrm{n}=6$ & $\mathrm{n}=6$ & $\mathrm{n}=6$ & $n=5$ & $n=5$ \\
\hline & $\begin{array}{c}4465 \text { (34) } \\
0.073 \\
(S D 0.166)\end{array}$ & $\begin{array}{c}630(\mathbf{0}) \\
0.192 \\
(S D 0.270)\end{array}$ & $\begin{array}{c}1770(\mathbf{0}) \\
0.166 \\
(\mathrm{SD} 0.311)\end{array}$ & $\begin{array}{c}253(\mathbf{0}) \\
0.194 \\
(\mathrm{SD} 0.317)\end{array}$ & $\begin{array}{c}253(\mathbf{0}) \\
0.121 \\
(\mathrm{SD} 0.270)\end{array}$ & $\begin{array}{c}378(0) \\
0.299 \\
(S D 0.344)\end{array}$ & $\begin{array}{c}741(0) \\
0.166 \\
(S D 0.244)\end{array}$ \\
\hline \multirow[t]{2}{*}{ NOS } & $n=16$ & $n=6$ & $n=5$ & $n=7$ & $\mathrm{n}=\mathbf{5}$ & $n=6$ & $n=5$ \\
\hline & $\begin{array}{c}4371(141) \\
0.200 \\
\text { (SD 0.206) }\end{array}$ & $\begin{array}{c}1326(\mathbf{0}) \\
0.198 \\
(S D 0.274)\end{array}$ & $\begin{array}{c}\mathbf{8 2 0}(\mathbf{0}) \\
0.282 \\
(S D 0.295)\end{array}$ & $\begin{array}{c}1770(\mathbf{0}) \\
0.172 \\
(S D 0.247)\end{array}$ & $\begin{array}{c}55(\mathbf{0}) \\
0.243 \\
(S D 0.292)\end{array}$ & $\begin{array}{c}990(0) \\
0.246 \\
(S D 0.289)\end{array}$ & $\begin{array}{c}351(\mathbf{0}) \\
0.356 \\
(S D 0.330)\end{array}$ \\
\hline \multirow[t]{2}{*}{ REL2 } & $n=16$ & $n=14$ & $n=10$ & $\mathrm{n}=6$ & $\mathrm{n}=\mathbf{8}$ & $n=7$ & $\mathrm{n}=\mathbf{8}$ \\
\hline & $\begin{array}{c}703(9) \\
0.073 \\
\text { (SD 0.175) }\end{array}$ & $\begin{array}{c}703(11) \\
0.143 \\
(S D 0.264)\end{array}$ & $\begin{array}{c}378(\mathbf{2}) \\
0.081 \\
(\mathrm{SD} 0.172)\end{array}$ & $\begin{array}{c}0(0) \\
\text { NA }\end{array}$ & $\begin{array}{c}21(\mathbf{0}) \\
0.577 \\
(S D 0.415)\end{array}$ & $\begin{array}{c}55(\mathbf{0}) \\
0.115 \\
(\mathrm{SD} 0.149)\end{array}$ & $\begin{array}{c}55 \mathbf{( 0 )} \\
0.091 \\
(\mathrm{SD} 0.207)\end{array}$ \\
\hline \multirow[t]{2}{*}{ FBN9 } & $n=16$ & $n=7$ & $\mathbf{n}=7$ & $\mathbf{n}=7$ & $n=7$ & $\mathrm{n}=7$ & $n=7$ \\
\hline & $\begin{array}{c}1378(16) \\
0.078 \\
(S D 0.175)\end{array}$ & $\begin{array}{c}780(0) \\
0.106 \\
(S D 0.156)\end{array}$ & $\begin{array}{c}\mathbf{1 0 8 1}(\mathbf{0}) \\
0.108 \\
(S D 0.206)\end{array}$ & $\begin{array}{c}190(\mathbf{0}) \\
0.766 \\
(S D 0.285)\end{array}$ & $\begin{array}{c}15(\mathbf{0}) \\
0.203 \\
(S D 0.264)\end{array}$ & $\begin{array}{c}66(\mathbf{0}) \\
0.231 \\
(S D 0.364)\end{array}$ & $\begin{array}{c}\mathbf{2 7 6}(\mathbf{0}) \\
0.103 \\
(S D 0.192)\end{array}$ \\
\hline
\end{tabular}

NA: no available data when sequences are not polymorphic. $n=$ number of individuals.

An. quadriannulatus, REL2 in An. melas and FBN9 in An. bwambae. These genes are not positioned in polymorphic chromosomal inversions in these species suggesting that the higher values of $r^{2}$ are not the result of limited recombination rates due to chromosomal arrangements. All other mean values are below 0.3, which reveals very limited LD taking into account the short distance between polymorphic sites (maximum distance between SNPs is 1176 bases). Indeed, compared to other species, the LD values observed here are among the lowest. Similar low LD levels were previously observed, mainly in plants [33-36]. Much higher LD is commonly observed in a wide range of species, for example cultivated and wild plants [37,38], birds [3] and mammals [39-43]. In Drosophila, LD often appeared to be higher than what we observed in Anopheles, but was also very variable depending on gene and genomic region [44-46] probably as a result of variable recombination rates and natural selection [47]. In our data, the comparison of mean $\mathrm{r}^{2}$ values in full sequences and sequence fragments revealed no significant differences between species or genes. This suggests that in different genomic regions, and members of the An. gambiae complex, LD in immunity genes, and probably others, is limited. However the small number of genes and chromosomal regions tested in the present study cannot allow conclusion for the whole An. gambiae genome as variations along the chromosomes and between mosquito populations are expected. In particular, the effect of chromosomal inversions was not tested here. The populations of An. gambiae s.s. (M and S) from Cameroon are known to be almost fixed for the standard chromosomal arrangements [48]. Testing the effect of the major chromosomal inversions of An. gambiae s.s. on LD would require sampling of populations polymorphic for these inversions, karyotyping the chromosomes and sequencing numerous genes inside and outside the inversions. Such a study would be of high interest as the resolution necessary for association studies could vary in chromosomal inversions or other genomic regions, and important genes involved in adaptation or controlling Plasmodium infection are expected to be located inside chromosomal inversions [22,49].

LD curves in $A n$. gambiae species show very fast decay for most of the tested genes and species. Generally, at very short nucleotide distances, less than 200 bases, LD decay curves were below an $r^{2}$ of 0.3 , although sporadic LD peaks were observed over distance. In exception to this is $A n$. bwambae FBN9 whose decay curve never falls as low as $\mathrm{r}^{2}=0.3$ in the 807 base region tested (Figure 1). The grid plots of $A n$. gambiae $\mathrm{M}$ form showed similar results with few pairwise estimates in LD. Interestingly some haploblocks were observed (in Gambicin and NOS) showing strong 


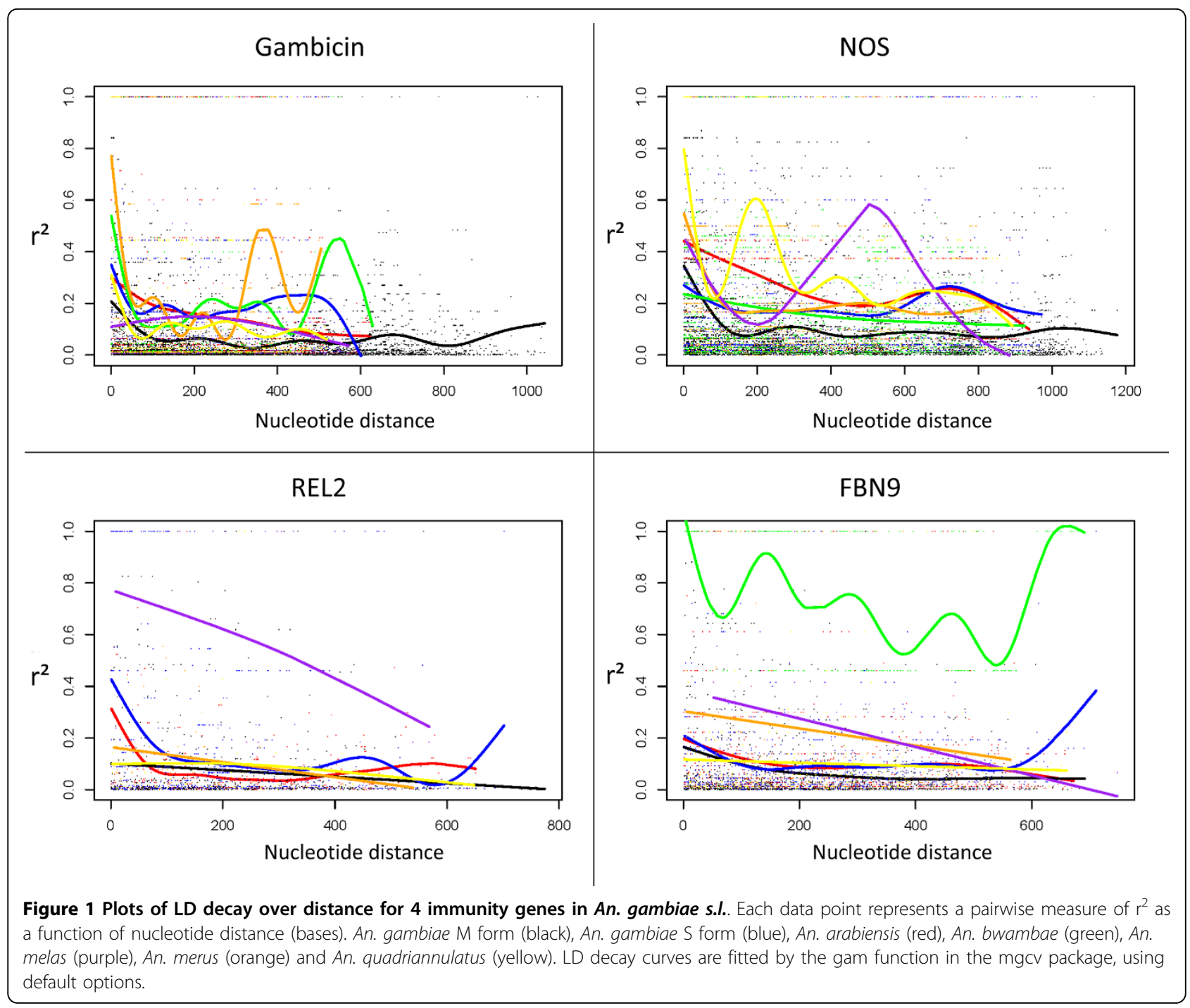

LD between markers. This pattern was observed only for very short distance markers, not more distant than 50 base pairs (Figure 2). This suggested variation in LD along the genes but a very limited extension of high LD blocks. In association studies, depending on the contribution of the causative allele on the observed phenotype and the sample size, a minimum threshold of $\mathrm{r}^{2} \geq 0.33$ to 0.8 can be used to consider whether SNPs above this limit with the causative SNP are potentially indicative of association $[47,50]$. This suggests that, for GWAS in An. gambiae s.l., very dense marker coverage will be required and the development of high-throughput genotyping tools essential for whole genome scans. However, in candidate gene association studies, a very high resolution genetic map can be more feasible by limiting genotyping to genes that have functional relevance. Also a rapid breakdown of LD will be favorable for identification of causative genes located in quantitative trait loci (QTL) by favoring high resolution mapping.

$\mathrm{LD}$ in a population is the result of various parameters, for example recombination and mutation rates, population structure and demographic history [47]. In species of the An. gambiae complex, their demographic history has been closely linked to anthropic changes [51] and their population sizes probably drastically increased some thousands of years ago. Rapid population growth decreases LD, and could be one of the reasons for the limited LD we observe here but could also be in part due to high mutation and recombination rates.

\section{Conclusion}

In this study, we observed limited LD in wild populations of seven species of the An. gambiae complex in four immunity genes. This suggests that GWAS will require a huge effort in genotyping and that a more 


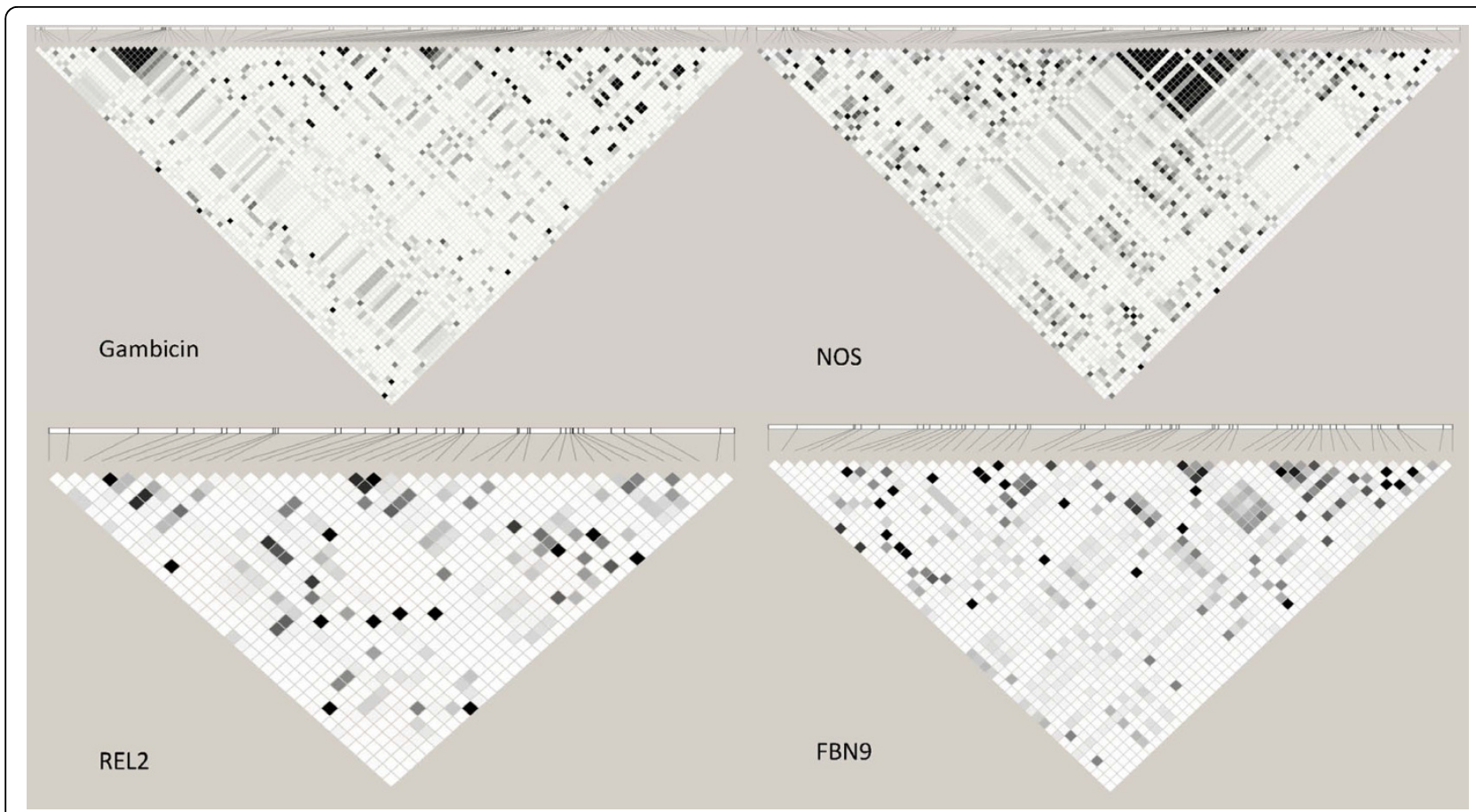

Figure 2 Grid plots for $\mathbf{4}$ immunity genes in An. gambiae $\mathbf{M}$ form. Each square corresponds to one LD value $\left(r^{2}\right)$ between 2 variant sites using a color code from a complete lack of $\operatorname{LD}\left(r^{2}=0\right.$ : white) to a perfect $L D\left(r^{2}=1\right.$ : black). Relative position of the SNPs is represented on the top line.

realistic approach might be to search for functional variations in putative candidate loci. On the other hand, the rapid decay of LD suggests that it will be possible to map functional variation at very fine scales in $A n$. gambiae s.l. populations. The present study however involved a limited number of chromosomal regions and populations so further work is needed to ascertain LD throughout An. gambiae genomes.

\section{Acknowledgements}

This work was funded by an ANR grant awarded to A.C.

\section{Author details}

'Laboratoire de Lutte Contre les Insectes Nuisibles, Unité de Recherche 016Institut de Recherche pour le Développement, B.P. 64501, 34394 Montpellier Cedex 5, France. ${ }^{2}$ Institut des Sciences de l'Évolution, Université Montpellier 2, CNRS, Place Eugène Bataillon, CC065, Montpellier, Cedex 5, France.

${ }^{3}$ Institut de Recherche pour le Développement, Institut de Recherche en Sciences de la Santé, Bobo Dioulasso, Burkina Faso.

\section{Authors' contributions}

A.C., I.M., F.R. and D.F. designed the study; C.H. carried out the experiments; A.C., C.H. and F.R. analysed the data; and A.C., C.H., F.R., I.M. and D.F. wrote the paper. All authors read and approved the final manuscript.

Received: 11 March 2010 Accepted: 15 September 2010 Published: 15 September 2010

\section{References}

1. Slatkin M: Linkage disequilibrium-understanding the evolutionary past and mapping the medical future. Nat Rev Genet 2008, 9:477-485.
2. LaFramboise $\mathrm{T}$ : Single nucleotide polymorphism arrays: a decade of biological, computational and technological advances. Nucleic Acids Res 2009, 37:4181-4193.

3. Backstrom N, Qvarnstrom A, Gustafsson L, Ellegren $H$ : Levels of linkage disequilibrium in a wild bird population. Biol Lett 2006, 2:435-438.

4. Gillies MT, De Meillon B: The Anophelinae of Africa South of the Sahara. Johannesburg, South Africa 1968.

5. Holt RA, Subramanian GM, Halpern A, Sutton GG, Charlab R, Nusskern DR, Wincker P, Clark AG, Ribeiro JM, Wides $R$, et al: The genome sequence of the malaria mosquito Anopheles gambiae. Science 2002, 298:129-149.

6. Vectorbase. [http://www.vectorbase.org/]

7. Cohuet A, Krishnakumar S, Simard F, Morlais I, Koutsos A, Fontenille D, Mindrinos M, Kafatos FC: SNP discovery and molecular evolution in Anopheles gambiae, with special emphasis on innate immune system. BMC Genomics 2008, 9:227.

8. Parmakelis A, Slotman MA, Marshall JC, Awono-Ambene PH, AntonioNkondjio C, Simard F, Caccone A, Powell JR: The molecular evolution of four anti-malarial immune genes in the Anopheles gambiae species complex. BMC Evol Biol 2008, 8:79.

9. Morlais I, Poncon N, Simard F, Cohuet A, Fontenille D: Intraspecific nucleotide variation in Anopheles gambiae: new insights into the biology of malaria vectors. Am J Trop Med Hyg 2004, 71:795-802.

10. Wilding CS, Weetman D, Steen K, Donnelly MJ: High, clustered, nucleotide diversity in the genome of Anopheles gambiae revealed through pooledtemplate sequencing: implications for high-throughput genotyping protocols. BMC Genomics 2009, 10:320.

11. Black WCt, Gorrochetegui-Escalante N, Randle NP, Donnelly MJ: The Yin and Yang of linkage disequilibrium: mapping of genes and nucleotides conferring insecticide resistance in insect disease vectors. Adv Exp Med Biol 2008, 627:71-83

12. White BJ, Cheng C, Simard F, Costantini C, Besansky NJ: Genetic association of physically unlinked islands of genomic divergence in incipient species of Anopheles gambiae. Mol Ecol 2010.

13. Ayala FJ, Coluzzi M: Chromosome speciation: humans, Drosophila, and mosquitoes. Proc Natl Acad Sci USA 2005, 102(Suppl 1):6535-6542. 
14. Stump AD, Shoener JA, Costantini C, Sagnon N, Besansky NJ: Sex-linked differentiation between incipient species of Anopheles gambiae. Genetics 2005, 169:1509-1519.

15. Slotman MA, Tripet F, Cornel AJ, Meneses CR, Lee $Y$, Reimer $L$, Thiemann TC, Fondjo E, Fofana A, Traore SF, Lanzaro GC: Evidence for subdivision within the M molecular form of Anopheles gambiae. Mol Ecol 2007, 16:639-649.

16. Lehmann T, Hawley WA, Grebert H, Collins FH: The effective population size of Anopheles gambiae in Kenya: implications for population structure. Mol Biol Evol 1998, 15:264-276.

17. Dong Y, Aguilar R, Xi Z, Warr E, Mongin E, Dimopoulos G: Anopheles gambiae immune responses to human and rodent Plasmodium parasite species. PLoS Pathog 2006, 2:e52.

18. Luckhart S, Vodovotz Y, Cui L, Rosenberg R, Cui LW: The mosquito Anopheles stephensi limits malaria parasite development with inducible synthesis of nitric oxide. Proceedings of the National Academy of Sciences of the United States of America 1998, 95:5700-5705.

19. Gupta L, Molina-Cruz A, Kumar S, Rodrigues J, Dixit R, Zamora RE, BarillasMury C: The STAT pathway mediates late-phase immunity against Plasmodium in the mosquito Anopheles gambiae. Cell Host Microbe 2009, 5:498-507.

20. Tahar R, Boudin C, Thiery I, Bourgouin C: Immune response of Anopheles gambiae to the early sporogonic stages of the human malaria parasite Plasmodium falciparum. EMBO J 2002, 21:6673-6680.

21. Garver LS, Dong Y, Dimopoulos G: Caspar controls resistance to Plasmodium falciparum in diverse anopheline species. PLoS Pathog 2009, 5:e1000335

22. Coluzzi M, Sabatini A, della Torre A, Di Deco MA, Petrarca V: A polytene chromosome analysis of the Anopheles gambiae species complex. Science 2002, 298:1415-1418.

23. Fanello C, Santolamazza F, della Torre A: Simultaneous identification of species and molecular forms of the Anopheles gambiae complex by PCRRFLP. Med Vet Entomol 2002, 16:461-464.

24. Kumar S, Nei M, Dudley J, Tamura K: MEGA: a biologist-centric software for evolutionary analysis of DNA and protein sequences. Brief Bioinform 2008, 9:299-306

25. Librado P, Rozas J: DnaSP v5: a software for comprehensive analysis of DNA polymorphism data. Bioinformatics 2009, 25:1451-1452.

26. R Development Core Team. [http://www.R-project.org].

27. Hill WG, Weir BS: Variances and covariances of squared linkage disequilibria in finite populations. Theor Popul Biol 1988, 33:54-78.

28. Hastie T, Tibshirani R, Friedman J: The elements of statistical learning: data mining, inference, and prediction. N. Y.: Springer 2009.

29. Barrett JC, Fry B, Maller J, Daly MJ: Haploview: analysis and visualization of LD and haplotype maps. Bioinformatics 2005, 21:263-265.

30. Simard F, Licht M, Besansky NJ, Lehmann T: Polymorphism at the defensin gene in the Anopheles gambiae complex: testing different selection hypotheses. Infect Genet Evol 2007, 7:285-292.

31. Little TJ, Cobbe N: The evolution of immune-related genes from disease carrying mosquitoes: diversity in a peptidoglycan- and a thioesterrecognizing protein. Insect Mol Biol 2005, 14:599-605.

32. Dassanayake RS, Silva Gunawardene YI, Tobe SS: Evolutionary selective trends of insect/mosquito antimicrobial defensin peptides containing cysteine-stabilized alpha/beta motifs. Peptides 2007, 28:62-75.

33. Brown GR, Gill GP, Kuntz RJ, Langley CH, Neale DB: Nucleotide diversity and linkage disequilibrium in loblolly pine. Proc Natl Acad Sci USA 2004, 101:15255-15260.

34. Remington DL, Thornsberry JM, Matsuoka Y, Wilson LM, Whitt SR, Doebley J, Kresovich S, Goodman MM, Buckler ESt: Structure of linkage disequilibrium and phenotypic associations in the maize genome. Proc Natl Acad Sci USA 2001, 98:11479-11484.

35. Xing Y, Frei U, Schejbel B, Asp T, Lubberstedt T: Nucleotide diversity and linkage disequilibrium in 11 expressed resistance candidate genes in Lolium perenne. BMC Plant Biol 2007, 7:43.

36. Ingvarsson PK: Nucleotide polymorphism and linkage disequilibrium within and among natural populations of European aspen (Populus tremula L., Salicaceae). Genetics 2005, 169:945-953.

37. Kolkman JM, Berry ST, Leon AJ, Slabaugh MB, Tang S, Gao W, Shintani DK, Burke JM, Knapp SJ: Single nucleotide polymorphisms and linkage disequilibrium in sunflower. Genetics 2007, 177:457-468.
38. Olson MS, Robertson AL, Takebayashi N, Silim S, Schroeder WR, Tiffin P: Nucleotide diversity and linkage disequilibrium in balsam poplar (Populus balsamifera). New Phytol 2010, 186:526-536.

39. Hernandez RD, Hubisz MJ, Wheeler DA, Smith DG, Ferguson B, Rogers J, Nazareth L, Indap A, Bourquin T, McPherson J, et al: Demographic histories and patterns of linkage disequilibrium in Chinese and Indian rhesus macaques. Science 2007, 316:240-243.

40. Amaral AJ, Megens HJ, Crooijmans RP, Heuven HC, Groenen MA: Linkage disequilibrium decay and haplotype block structure in the pig. Genetics 2008, 179:569-579.

41. Hinds DA, Stuve LL, Nilsen GB, Halperin E, Eskin E, Ballinger DG, Frazer KA, Cox DR: Whole-genome patterns of common DNA variation in three human populations. Science 2005, 307:1072-1079.

42. Long JR, Zhao LJ, Liu PY, Lu Y, Dvornyk V, Shen H, Liu YJ, Zhang YY, Xiong DH, Xiao P, Deng HW: Patterns of linkage disequilibrium and haplotype distribution in disease candidate genes. BMC Genet 2004, 5:11.

43. Laurie CC, Nickerson DA, Anderson AD, Weir BS, Livingston RJ, Dean MD, Smith KL, Schadt EE, Nachman MW: Linkage disequilibrium in wild mice. PLoS Genet 2007, 3:e144.

44. Schaeffer SW, Goetting-Minesky MP, Kovacevic M, Peoples JR, Graybill JL, Miller JM, Kim K, Nelson JG, Anderson WW: Evolutionary genomics of inversions in Drosophila pseudoobscura: evidence for epistasis. Proc Natl Acad Sci USA 2003, 100:8319-8324.

45. Wang W, Thornton K, Emerson JJ, Long M: Nucleotide variation and recombination along the fourth chromosome in Drosophila simulans. Genetics 2004, 166:1783-1794.

46. Schlenke TA, Begun DJ: Linkage disequilibrium and recent selection at three immunity receptor loci in Drosophila simulans. Genetics 2005, 169:2013-2022.

47. Ardlie $K G$, Kruglyak $L$, Seielstad M: Patterns of linkage disequilibrium in the human genome. Nat Rev Genet 2002, 3:299-309.

48. Wondji C, Frederic S, Petrarca V, Etang J, Santolamazza F, Della Torre A, Fontenille D: Species and populations of the Anopheles gambiae complex in Cameroon with special emphasis on chromosomal and molecular forms of Anopheles gambiae s.s. J Med Entomol 2005, 42:998-1005.

49. Riehle MM, Markianos K, Niare O, Xu J, Li J, Toure AM, Podiougou B, Oduol F, Diawara S, Diallo M, et al: Natural malaria infection in Anopheles gambiae is regulated by a single genomic control region. Science 2006, 312:577-579.

50. Carlson CS, Eberle MA, Rieder MJ, Yi Q, Kruglyak L, Nickerson DA: Selecting a maximally informative set of single-nucleotide polymorphisms for association analyses using linkage disequilibrium. Am J Hum Genet 2004, 74:106-120.

51. Hume JC, Lyons EJ, Day KP: Human migration, mosquitoes and the evolution of Plasmodium falciparum. Trends Parasitol 2003, 19:144-149.

doi:10.1186/1471-2156-11-81

Cite this article as: Harris et al.: Low linkage disequilibrium in wild Anopheles gambiae s.l. populations. BMC Genetics 2010 11:81.

\section{Submit your next manuscript to BioMed Central and take full advantage of:}

- Convenient online submission

- Thorough peer review

- No space constraints or color figure charges

- Immediate publication on acceptance

- Inclusion in PubMed, CAS, Scopus and Google Scholar

- Research which is freely available for redistribution

Submit your manuscript at www.biomedcentral.com/submit
C Biomed Central 